\title{
Coulisses
}

Revue de théâtre

1 | Printemps 1990

Varia

\section{Le théâtre spontané comme laboratoire de transformation sociale}

Jean-François de Raymond

\section{(2) OpenEdition}

1 Journals

Édition électronique

URL : http://journals.openedition.org/coulisses/1566

DOI : $10.4000 /$ coulisses. 1566

ISSN : 2546-9460

Éditeur

Presses universitaires de Franche-Comté

Édition imprimée

Date de publication : 1 avril 1990

Pagination : 67-70

ISSN : 1150-594X

\section{Référence électronique}

Jean-François de Raymond, "Le théâtre spontané comme laboratoire de transformation sociale »,

Coulisses [En ligne], 1 | Printemps 1990, mis en ligne le 04 juillet 2017, consulté le 23 octobre 2019.

URL : http://journals.openedition.org/coulisses/1566; DOI : 10.4000/coulisses. 1566

Ce document a été généré automatiquement le 23 octobre 2019.

Coulisses 


\title{
Le théâtre spontané comme laboratoire de transformation sociale
}

\author{
Jean-François de Raymond
}

\section{NOTE DE L'ÉDITEUR}

Reproduit avec l'aimable autorisation des Annales littéraires de l'université de Besançon, in Actes du colloque Théâtre et Révolution, p. 205.

1 C'est dans l'art contemporain que l'improvisation a été pour la première fois réhabilitée. Au théâtre d'abord, avec Stanislawski et Jacques Copeau, dont les méthodes ont été souvent reprises depuis, que ce soit par Grotowski, l'Actor's Studio ou le Living Théâtre. Dans le domaine musical, ensuite, grâce au jazz, bien sûr, mais aussi à l'utilisation de plus en plus fréquente de procédures aléatoires par des compositeurs comme Boulez, Stockhausen, John Cage ou les techniciens de la musique électroacoustique. Dans le domaine pictural, enfin, avec l'Action painting chère à Jackson Pollock et à tout le mouvement expressionniste abstrait.

2 L'improvisateur a choisi de se livrer à l'avenir en épousant le présent, ou de vivre pleinement le présent pour ne pas se laisser détruire par le passé. Môme s'il n'est pas toujours et en tout lieu un improvisateur, môme s'il distingue l'improvisation (bonne) et l'impréparation (mauvaise), même s'il sait parfaitement qu'improviser s'apprend, il sait aussi qu'apprendre à improviser, c'est apprendre à revivre. Comme tout un chacun, il est pris dans le temps; mais chaque fois qu'il improvise, il éprouve le sentiment de fonder ce temps dans lequel il est pris, bref de redevenir le contemporain des origines.

L'improvisation s'exerce aussi dans d'autres domaines: placés dans une situation d'urgence, le chirurgien ou le stratège doivent être capables d'improviser - c'est même dans ces cas-là que leur maîtrise éclate. Il n'en va pas autrement pour l'homme politique : la principale qualité de ce perpétuel improvisateur n'est-elle pas la capacité 
de réagir immédiatement à l'événement imprévu, d'épouser complètement le problème qui se présente à l'instant même où il se pose?

4 Les foules, elles aussi, improvisent : qu'il s'agisse du carnaval et de la fête d'une part, ou de cette fête qui tourne mal et qu'on appelle l'émeute d'autre part, il est clair que l'improvisation est l'une des dimensions fondamentales du social.

5 Or, cette improvisation ${ }^{1}$ que notre siècle a partiellement reconnue, trouve en Jacob Lévi Moreno le praticien de la spontanéité et son prophète : Who shall survive?

6 Dans Who shall survive ${ }^{2}$, Moreno se présente après Pinel qui délivra les malades de leurs chaînes et Freud qui libéra le psychisme des tyrannies de l'inconscient comme l'auteur de la troisième révolution psychiatrique qui inventa la thérapie de groupe, la sociométrie et le psychodrame.

7 Le théâtre spontané relate la genèse de cette " troisième révolution »: il se situe entre les ouvrages de technique thérapeutique et de philosophie sociale de Moreno qu'il annonce par son côté expérimental, et les premiers écrits dont il tient sa conception judéo-métaphysique de la cosmogénèse.

8 Dans cet ouvrage se dessinent les prémisses de la sociométrie, de la théorie cosmogénétique "qui dépassera théoriquement le positivisme d'Auguste Comte et la théorie révolutionnaire de la praxis de Karl Marx », et la première présentation du psychodrame et de ses fondements. Attestant la fécondité des premières intuitions moréniennes où s'ébauchent les linéaments des techniques développées dans les ouvrages ultérieurs, le théâtre spontané se révèle comme une plaque tournante dans l'œuvre de Moreno.

9 Le théâtre spontané, créé à Vienne, passait pour révolutionnaire: plus d'auteur, de texte, ni de distinction entre acteurs et public, la création devient l'affaire de tous, simultanément, ici et maintenant. Cette tentative eut un retentissement sur le théâtre américain peu après l'immigration de Moreno aux USA en 1925. Le théâtre contemporain a assimilé les découvertes moréniennes mais en un sens plus timide qu'on ne le croirait : il ne se confie que par fragments à l'aventure de la spontanéité, ses improvisations restent sectorielles : en fait, personne n'est allé aussi loin que Moreno.

10 Par différence avec le théâtre traditionnel, de répétition, et ses « conserves culturelles ${ }^{3}$ » où le rapport temporel est le trait d'union entre un écrit passé et la représentation présente qu'il commande, le théâtre spontané est un théâtre d'animation dont le rapport temporel est ponctuel : l'instantanéité de l'ici et maintenant. Dans le premier cas il y a séparation entre le temps homogène, réglé, différé, de la préparation, de la nature et celui de la représentation, calque du premier, dont l'idéal est de le répéter sans y rien changer ; cette situation de succession exclut l'engagement de l'acteur. Dans le second il $\mathrm{y} \mathrm{a}$, au contraire, unité dans le temps discontinu, hétérogène et imprévisible du jeu, sans répétition préalable; la situation est celle de la simultanéité avec l'engagement de chaque actant, contemporain de celui de l'autre en sa spontanéité.

11 Thème essentiel de la pensée de Moreno la spontanéité constitue la réponse adaptée à un situation nouvelle ou originale par rapport à une situation ancienne ; la spontanéité varie entre le réflexe et la réflexion. En fait, la spontanéité motrice est associée à la créativité: la créativité sans la spontanéité reste sans vie, la spontanéité sans la créativité est vide et stérile. Chacun risquerait d'être "un idiot spontané » ou «un créateur désarmé ». Mais c'est moins la créativité qui distingue les hommes les uns des 
autres (beaucoup sont doués et seraient aptes à la création alors que peu créent effectivement), que la spontanéité, car celle-ci «fait passer à l'acte les aptitudes virtuelles ». La spontanéité a un pouvoir d'effectivité : elle incite l'individu capable de création, elle permet la socio-création à partir de l'acte selon lequel les personnes vont les unes vers les autres et aboutissent à des interactions constitutives de groupes. La spontanéité anime la créativité, qui lui offre un contenu et une occasion.

L'idée initiale de Moreno fut celle de l'existence d'un moi spontanément créateur avec les risques d'éparpillement d'une spontanéité non contrôlée. Relative selon les individus, la spontanéité est en fait assez rare et souvent atrophiée : elle est en raison inverse de l'organisation. Richesse du pauvre, procédé du démuni, la spontanéité ne laisse jamais dépourvu celui qui s'y confie, au contraire de la mémoire qui trahit parfois en le plongeant dans l'embarras, l'acteur régulier dont la spontanéité est oblitérée par les clichés qui s'y sont substitués.

Le point de départ de l'état spontané est réchauffement : vécu comme pleinement libre par le sujet qui l'expérimente, il conduit à l'acte spontané. Il faut donc s'y exercer. À la répétition correspond ici l'entraînement; la valeur de la spontanéité ne lui vient pas seulement du présent mais aussi de l'avenir, c'est aussi une valeur pédagogique. L'acteur comme tout atome social spontané n'a plus seulement à savoir des rôles fixes mais à bien se connaître, la pédagogie, substitut de la répétition, consistant ici à associer vie (communautaire) et jeu (improvisé), le théâtre comme chez d'autres praticiens radicaux, reconduit au social. Cette pédagogie toute active porte moins sur les méthodes de reproduction que sur la provocation à l'existence personnelle dans toutes les situations de la vie sociale ; apprendre à libérer sa spontanéité, ce n'est pas apprendre des contenus, des actes tout prêts à reproduire mais cela se conquiert selon les exigences de la réalité.

Son apprentissage, toujours urgent, l'est d'autant plus qu'elle risque toujours de se congeler en rôles fixes qui répètent des formules, comme le théâtre spontané en Commedia dell'Arte.

L'histoire de l'espèce est celle d'un enkystement de la spontanéité : le primitif voulut conserver; « les conserves de la technique et de la culture » qu'il élabora réduisirent sa créativité et sa spontanéité. L'enfant perd moins vite sa spontanéité que l'adulte car il doit s'adapter pour réussir et sa spontanéité reste la plupart du temps en action: proche du status nascendi de ses expériences, l'enfant n'est pas encore fixé dans des stéréotypes culturels, comme l'avait déjà noté Bergson dont la perspective sur la spontanéité a tant de points communs avec celle de Moreno.

Souvent inhibée ou recouverte par des conserves ou des mécanismes culturels, l'insuffisance du développement de la spontanéité est responsable de nombreux troubles.

17 Ces recherches trouvèrent une application dans la thérapeutique psychologique. On sait la parenté avec le jeu dramatique (rappelée par le théâtre lui-même avec MaratSade de Peter Weiss) les troubles et les conflits constituant la difficulté existentielle s'améliorent effectivement par leur mise en scène vécue. Ainsi pour redevenir vivable, le réel exige un passage par l'imaginaire comme par ce qui ressource une conduite appauvrie par un seul possible stéréotypé, à une pluralité enrichissante. La réalité se ressource au rêve et le réel aux possibles comme à leur matrice, chaque actant devient à lui-même son propre poète et ainsi retrouve en lui la création dont il avait perdu la capacité. En effet, moins développée que les facultés intellectuelles, la spontanéité 
connaît des flambées, mais finit par se plier aux impératifs des stéréotypes sociaux et culturels.

C'est cependant la grande force d'indétermination car elle sauve l'imprévisible en l'homme, irréductible à tout apprentissage comme à toute transmission elle échappe à la loi de conservation de l'énergie : ni quantifiée ni mesurable, on ne peut la conserver en réserve dans des accumulateurs. Elle ne fonctionne qu'au moment où elle jaillit; au contraire des conserves culturelles, éphémère, c'est pourquoi elle anime la créativité, dynamisme du commencement. Or, note Moreno, nous vivons le temps où l'homme, disposant d'un pouvoir décuplé par ses machines, peut se livrer à sa spontanéité et " expérimenter sur la spontanéité elle-même", ouvrant, dès lors, les perspectives fécondes.

ouvre ainsi de nouvelles dimensions en appliquant aux sociétés l'analyse de la spontanéité : celle-ci anime une forme, un rôle, toujours social. Elle concourt à l'adéquation, toujours problématique, de ce rôle en ses exigences socio-culturelles et de la spontanéité d'une personne en ses particularités spatio-temporelles - tout rôle ne correspondant pas nécessairement à la spontanéité de chaque personne à un moment donné et chacun ne se sentant pas nécessairement motivé par n'importe quel rôle. À cette question - politique - de répartition ou de gestion, en termes philosophiques, celle de la vocation, la spontanéité ouvre des perspectives.

Moreno a bien vu, ce qui fait l'accent si actuel de sa pensée, le danger de paralysie qui guette toute institution et comme cette détermination objective appelle le recours à une vivante spontanéité.

La perspective de Moreno s'ouvre sur l'humanité dans sa totalité : «Who shall survive?» demande l'auteur qui différencie sa démarche par rapport aux idées chrétiennes, à celles de Marx, de Nietzsche et de Freud, par son projet thérapeutique visant à guérir, à rendre possible une existence sociale épanouissante pour chacun, (en laissant l'individu " suivre ses inclinations naturelles et rallier spontanément les groupes qui l'attirent ${ }^{4} »$ ). À cette question prophétique sur l'avenir dont nous trouvons les linéaments dans le théâtre spontané avec l'évocation de la menace atomique et de la désagrégation culturelle, la réponse consiste précisément dans l'apprentissage de la liberté et de la création sur le mode social, « construire une société où chaque membre serait appelé à participer librement à la formation des groupes dont il fait partie, où les différents groupes qui la constituent, seraient si bien organisés, si bien adaptés les uns aux autres qu'ils formeraient une république durable et harmonieuse » $(\mathrm{Td})$. Cette "révolution sociale expérimentale " se trouve en germes dans le théâtre spontané où Moreno révèle son objectif (théorique) : saisir en l'observant, le processus de changement in statu nascendi et l'objectif (pratique) : l'intervention psychologique. Il situe la révolution dite "sociométrique " - à réaliser - au sommet des autres révolutions car son efficacité atteindrait un résultat en profondeur qu'elles n'ont pu atteindre: elle réduirait le "prolétariat affectif».

Il ne s'agit d'ailleurs que de la réanimation actuelle d'une force engourdie ou affaiblie en suivant « la direction d'une évolution spontanée et créatrice, la direction même de la vie et du temps ${ }^{5} »$. On entrevoit les transformations qui en découleraient si les "nécessités " sociales ne l'étouffaient pas, si elle n'était pas considérée comme une aberration mais comme le dynamisme contagieux capable de mobiliser les masses et de renverser les montagnes. Moreno prévoit là une source inimaginable d'énergie sociale ${ }^{6}$. 

l'exécution et la conception - ce qui pose autrement le problème des rôles puisqu'ils construisent la personnalité et renvoie à une philosophie politique concernant la détention du pouvoir dans la cité.

24 la spontanéité (manifeste-la et tu la trouveras chez autrui), la sincérité (et elle sera donnée aussi), l'amour (et le groupe le manifestera) - «la sociométrie est l'éthique sociale par excellence ».

25 Nous avons vu la solidification du spontané en conservé ; d'autre part rien qui atteigne le centre affectif de la personnalité ne peut se réaliser hors de la spontanéité et de la créativité dans les sociétés comme chez les individus : alors la continuité peut-elle accueillir la spontanéité sans la désarmorcer? Peut-on maîtriser la spontanéité pour qu'elle transforme durablement l'existence sociale? Ou peut-on élaborer systématiquement une "stratégie de création ", qui, selon l'expression de Moreno, permette de faire de l'humanité une association où la création comme résultat ne viendra plus faire obstacle à l'acte de création?

\section{NOTES}

1. Cf. Jean-François de Raymond, L'improvision. Contribution à la philosophie de l'action, Vrin, 1980.

2. Titre français prosaïque : Les Fondements de la Sociométrie, PUF : The Theatre of Spontaneity, trad. J.-F. de Raymond, présentation par J. Dumur, introduction par A. Schustenberger, Epi, 1985.

3. Moreno ne méprise pas les "conserves culturelles ", il les situe comme des pièces de musée dont la vie s'est envolée et qui ne peuvent prétendre être le champ d'une spontanéité vécue immédiate.

4. Fondements de la sociométrie, p. 3.

5. Ibid., p. 404.

6. Ibid., p. 75. 